\title{
UTILITY OPTIMIZATION FOR THE BEAM ORBIT STABILITY AT SRRC
}

\author{
H. M. Cheng", C. R. Chen, Z. D. Tsai, and J. R. Chen" \\ Synchrotron Radiation Research Center, Taiwan, R.O.C. \\ also at "Department of Nuclear Science, National Tsing-Hua University, Taiwan, R.O.C.
}

\section{Abstract}

At the Taiwan Light Source (TLS), the stability of the electron beam orbit is sensitive to the utility conditions. The beam position showed strong correlation with the temperature while the tunnel temperature variation was more than $0.4^{\circ} \mathrm{C}$. In this work, the coolant temperature and the air temperature in the storage ring tunnel were tuned to test the sensitivity of the electron beam orbit. The temperature difference of the devices in the TLS might be as high as $10^{\circ} \mathrm{C}$ between the system shutdown and operation. These temperature variations affected the beam stability through some specific routes. Some modeling technique was also applied to identify the correlation factors among the beam position and the utility conditions.

\section{INTRODUCTION}

In 1997, Keller et al. [1] studied the correlation between the beam orbit stability and the utility conditions for the Advanced Light Source (ALS). At TLS, a series of experiments were also conducted [2]. From the daily observation of the beam status at TLS, the beam orbit is sensitive to the utility conditions. Although an orbitfeedback system is being implemented at TLS, the requirement for a stable utility system should never be ignored. It is easy to imagine that the positions of beam position monitors, which used for orbit feedback, would follow the tiny thermal distortions.

To study the propagation routes caused by the utility perturbation, experiments have been setup. In this work, the experiments concentrated on thermal effects from the temperature varying devices. In the second section, a simulation model and the results for optimizing the control parameters of the utilities is mentioned. Instead of using linear modeling approach, a fuzzy modeling technique was applied to acquire the beamposition/utility-system model. In the third section, the optimization for deionized water (DIW) temperature setting is described.

\section{UTILITY SYSTEM AND MODELING}

The TLS utility consists of three major systems: the water system, the air conditioning system, and the electricity system. In this work, only the studies on the temperature varying systems are mentioned.

"Email: hmcheng@ @srrc.gov.tw

\subsection{Temperature Varying Devices}

The devices that might cause thermal variations are the cooling water system and the air-conditioning system. The cooling water system consists of three subsystems: the chilled water system (CHW), the cooling tower water system (CTW), and the de-ionized water system (DIW). The de-ionized water system consists of three loops. The DIW system-1 $(\mathrm{Cu})$ supplies the coolant to the major devices, such as the RF systems, the magnets, and the power supplies. The DIW system-2 (Al) is used for the vacuum beam ducts. The DIW system-3 (Beamline) supplies to the photon-beamline devices.

The air-conditioning system of the storage ring includes five major air-handling units supplying to the storage ring, the laboratory, and the experimental area. In addition, the air-conditioning system also supplies to the core area devices, at where the RF transmitters, magnet power supplies, vacuum controllers, and other associated instruments are located. Table 1 lists the current set points and the varying temperature range of the TLS utility systems.

Table 1: Operation temperature of the TLS utility system.

\begin{tabular}{ccccccc}
\hline \hline System & CHW & CTW & DIW $(\mathrm{Cu})$ & DIW $(\mathrm{Al})$ & $\mathrm{A} / \mathrm{C}$ \\
\hline \hline Set Point & $7.0^{\circ} \mathrm{C}$ & $30.0^{\circ} \mathrm{C}$ & $23.5^{\circ} \mathrm{C}$ & $25.0^{\circ} \mathrm{C}$ & $24.5^{\circ} \mathrm{C}$ \\
Range & $\pm 0.2^{\circ} \mathrm{C}$ & $\sim \pm 0.5^{\circ} \mathrm{C}$ & $\pm 0.2^{\circ} \mathrm{C}$ & $\pm 0.2^{\circ} \mathrm{C}$ & $\pm 0.1^{\circ} \mathrm{C}$ \\
\hline
\end{tabular}

\subsection{Monitoring Systems}

The beam orbit was monitored by the electron beam position monitors (EBPM, $\approx 5 \mu \mathrm{m}$ resolution) and the photon beam position monitors (PBPM, with resolution $<0.5 \mu \mathrm{m})$. The temperature sensors at the coolant loop and in the ring tunnel monitored the utility performance. Most part of the utility system is using temperature sensors with $0.1^{\circ} \mathrm{C}$ sensitivity and part with $0.03^{\circ} \mathrm{C}$ sensitivity.

\subsection{System Modeling}

Figure 1 shows the possible transferring routes to the electron beam and photon beam perturbation from utility systems. The effects among the subsystems are cascades. In this work, the system modeling approach was studied for optimizing the utility status.

Based on the propagation routes, a mathematical model was established to calculate the orbit for a given set of 
operating parameters. The simulation code representing the TLS storage ring was written in MATLAB.

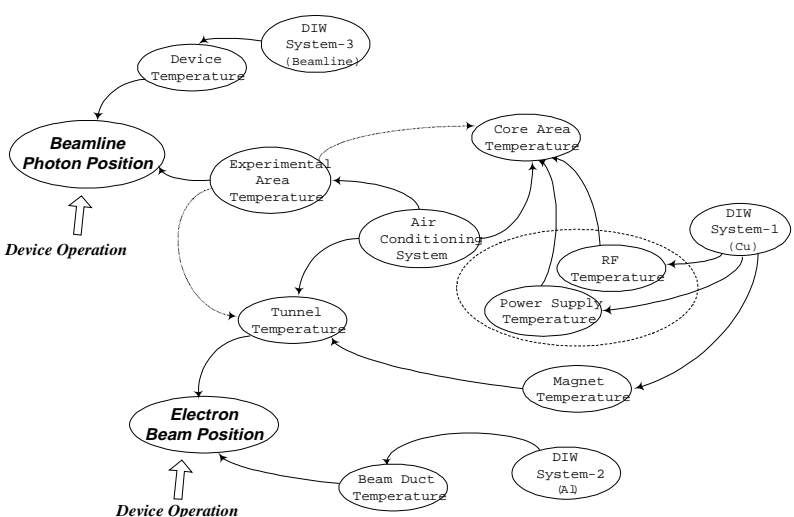

Figure 1: The propagation routes from the utility variation to the variations of electron- and photon-beam position.

The mathematical model of a linear time-invariant (LTI) system was described as:

state equation: $\frac{d \mathbf{X}_{\text {Status }}}{d t}=\mathbf{A} \mathbf{X}_{\text {Status }}+\mathbf{B} \mathbf{U}_{\text {Util }}+\mathbf{F w}$, and

output equation: $\mathbf{Y}_{\text {BPS }}=\mathbf{D} \mathbf{X}_{\text {Status }}+\mathbf{E} \mathbf{U}_{\text {Util }}+\mathbf{H w}$,

where $\mathbf{Y}_{\mathrm{BPS}}$ is the beam position, $\mathbf{X}_{\mathrm{Status}}$ is the temperature status vector at the storage ring, $\mathbf{U}_{\mathrm{Util}}$ is the set points vector of the utility systems, $\mathbf{w}$ is the disturbance vector, and $\mathbf{A}, \mathbf{B}, \mathbf{D}, \mathbf{E}, \mathbf{F}$ and $\mathbf{H}$ are the coefficient matrices with constant elements. For an LTI system $\mathbf{w}$ is always a null vector. In real life, the disturbance term always contains the nonlinearity of system fluctuation, invalid measurement, or the external unpredictable signals. In this work, the output is the EBPM results along the $y$-axis. The elements chosen in the input vector are the ambient temperatures around the kicker and the RF cavity in the tunnel, the outlet temperature of the DIW system-1 $(\mathrm{Cu})$ and system-2 (Al), and the temperature of the quadrupole magnet cable. The input data are shown in Figure 2a.

As a LTI system, the ARMAV [3] modeling approach was proposed. The standard deviation $\sigma$ of the calculating error (the difference between the measured and the calculated results) is about $18 \mu \mathrm{m}$ (Table 2 ).

To predict the effect caused by the nonlinear term, the fuzzy modeling approach proposed by M. Sugeno [4] was applied to build the simulation model in this work. Assuming that the beam position in the storage ring is only affected by the utility conditions, the data points shall locate in the $\mathrm{s}+1$ dimensional space $\left(\mathbf{X}^{(i)}, y^{(i)}\right)$, where $\mathbf{X}^{(i)}=$ $\left(x_{1}^{(i)}, x_{2}^{(i)}, \ldots x_{s}^{(i)}\right), x_{j}^{(i)}$ denotes the $j$-th (sensor index) input's at the $i$-th (time) point, and $y^{(i)}$ is the output's (beam position) coordinate of the $i$-th point. In the simulation result, the inputs are the same as the LTI modeling approach. The standard deviation of the calculation error is about $8 \mu \mathrm{m}$.

The nonlinear simulation procedure was applied to observe the performance of the generated model. Figure $2 \mathrm{~b}$ illustrates the simulation results of Sugeno's fuzzy model. In Table 2, the performance of the LTI model and the Sugeno's fuzzy model is compared.
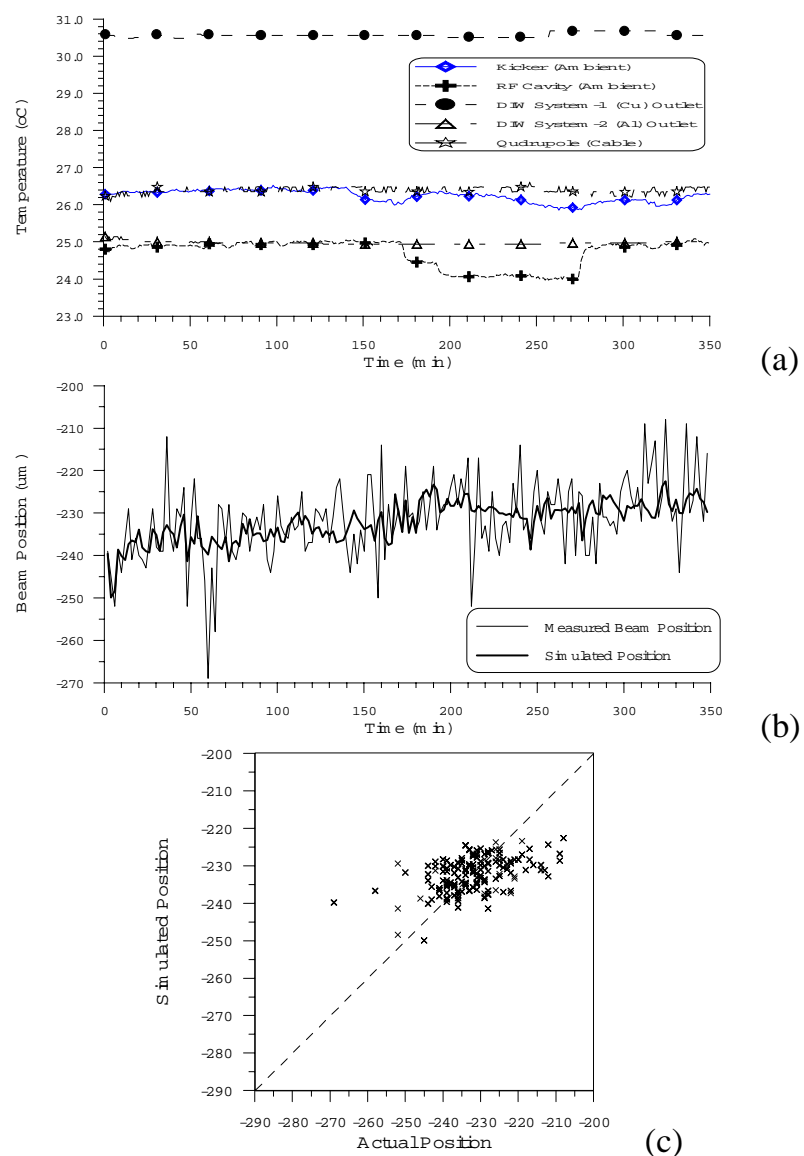

Figure 2: (a) Measured temperature inputs; (b) measured beam position and the simulation results of the fuzzy model; and (c) the statistics between the measured and simulated beam position.

In this simulation, the trend of the simulation results meet quite good with the measured data (Figure 2b). However, some parts of the simulation output do not meet the result (for example, the discrepancies at $\mathrm{t} \approx 60,120$-150, 210240, 260-280, 320, etc.). For improving, the model could be modified by two approaches. The first method is to divide the whole process into several different modes (e.g. transient mode, steady state mode, high/low current, etc.). The other method is to include more modeling parameters, such as mechanical vibration and electricity fluctuations.

Table 2: Standard deviation comparison of calculating error between ARMAV and Sugeno's fuzzy model.

\begin{tabular}{ccc}
\hline \hline & Linear (ARMAV) & Nonlinear (Sugeno) \\
\hline \hline$\sigma$ & $\sim 18 \mu \mathrm{m}$ & $\sim 8 \mu \mathrm{m}$ \\
\hline
\end{tabular}

In Figure 2c, the comparison of the results shows the performance of the model. As stated in Table 2, the standard deviation of the error is $\sim 8 \mu \mathrm{m}$. 


\section{TEMPERATURE SETTING OPTIMIZATION}

A series of experiment was conducted to test the relationships between the beam orbit variation and the utility parameters. The experimental results showed that some of the orbit oscillations were correlated to the temperature variation while the variation was larger than $0.4^{\circ} \mathrm{C}$.

At TLS, the utility capacity might saturate because of improper settings. The saturation conditions of the utility system were mainly determined by the DIW system-1. When the temperature set point of DIW system-1 is below $25.0^{\circ} \mathrm{C}$, the air-conditioning system is capable to keep the air temperature variation within $\pm 0.2^{\circ} \mathrm{C}$, and the beam orbit drift is therefore improved. If the set point falls beyond this range, some air-conditioning subsystems saturate. For example, if the set point is $25.0^{\circ} \mathrm{C}$, the AHU duty factor is $100 \%$ and saturates. Table 3 lists the relationship between the AHU duty factor and the temperature set point of DIW system-1.

Two major methods were adopted to stabilize the beam position in order to keep the utility systems unsaturated and to achieve an optimized balance. The first one was to optimize the set points for individual subsystems. Figure 4 shows the correlation between the beam drift in one shift and the DIW system-1 set point. It shows that the lower the DIW temperature is, the smaller the air temperature variation will be, and thus the smaller beam orbit variation occurs.

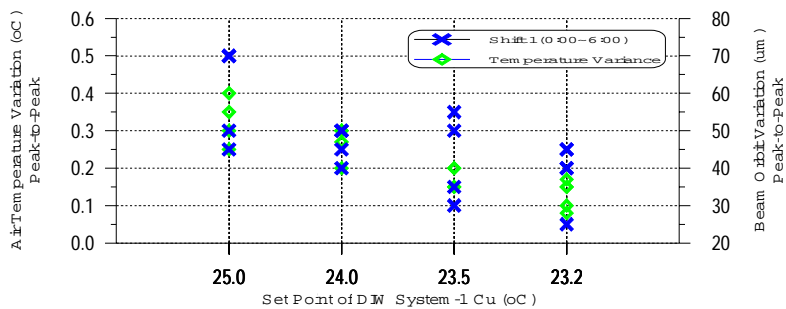

Figure 4: Relationship among the beam orbit variation, the air temperature, and the copper DIW temperature.

The second method was to improve the temperature shock in the air-conditioned area covered by two different AHUs. This kind of shock might be caused by an asynchronous control command or signal feedback. By following independent responses, sudden changes might occur between two separated AHUs. Instead of controlling the temperature of the storage ring with individual feedback, all the controllers in the ring follow the average temperature readout for feedback signal.

Table 3: Relationship between the DIW system-1 set points and the AHU duty factors.

\begin{tabular}{cccccc}
\hline \hline DIW set point $\left({ }^{\circ} \mathrm{C}\right)$ & 25.0 & 24.0 & 23.5 & 23.2 & 23.0 \\
\hline \hline Duty Factor & Saturated & $\sim 80 \%$ & $\sim 50 \%$ & $\sim 25 \%$ & Saturated \\
\hline
\end{tabular}

\section{DISCUSSION AND CONCLUSION}

Currently, the air temperature in the storage ring tunnel was held within $\pm 0.1^{\circ} \mathrm{C}$, and the cooling water system could also hold the outlet temperature within $\pm 0.2^{\circ} \mathrm{C}$. The source capacity of both air-handling units and the DIW system achieved a balance without saturation. After the improvements, the beam orbit variation was controlled to be less than $\pm 15 \mu \mathrm{m}$.

Though a modeling tool has been developed to correlate the beam position and the utility system, some ambiguities need to be resolved. Three major factors are to be considered for the improvement. The first factor is inhomogeneous air temperature in the storage ring tunnel. The second factor is the injection process between two shifts. The third, probably the most important factor, is the insufficient input parameters.

To solve these problems, some projects are underway. The injection energy is to be increased from $1.3 \mathrm{GeV}$ to $1.5 \mathrm{GeV}$ (so that no ramping process during injection) in order to overcome the transient phenomenon after injection. A series of experiments are being conducted to analyze the inhomogeneous environment so that the parameters for temperature control system can be better tuned. Some environmental factors, such as the mechanical vibration, the decreasing beam current, and the electricity perturbations, etc., will be added to make the model more complete. The improvement on modeling will help the optimization of the utility set points.

\section{ACKNOWLEDGEMENT}

The authors would like to thank to their colleagues in the utility group of TLS for their assistance. Thanks are also to the TLS operators for their engagement and help in all phases of the investigations.

\section{REFERENCES}

[1]R. Keller, and H. Nishimura, "Orbit Stability of the ALS Storage Ring,", Vancouver (1997).

[2]J.R. Chen, H.M. Cheng, C.R. Chen, Z.D. Tsai, G.Y Hsiun, and T.F. Lin, "The Correlation between Beam Orbit Stability and the Utilities at SRRC", Stockholm (1998).

[3]S.M. Pandit, and S.M. Wu, Time Series and System Analysis with Applications, John Wiley (1983).

[4]M. Sugeno, and T. Tanaka, "A Fuzzy Logic Based Approach to Qualitative Modeling”, IEEE Trans. Fuzzy Systems, vol. 1, no. 1, pp.7-31, 1993.

[5]W.H. Hsieh, and C.C. Cheng, "A New Approach to Complex Systems Modeling via Fuzzy Crystallization Algorithm and Its Application to Precision Insertion Operation”, Kaohsiung (1995). 